\title{
Can Chess Ever Be Solved
}

\author{
Naveen Kumar' ${ }^{1}$, Bhayaa Sharma ${ }^{2}$ \\ ${ }^{1,2}$ Department of Mathematics, University Institute of Sciences, Chandigarh University, Gharuan, Mohali, \\ Punjab-140413, India \\ 1imnaveenphd@gmail.com, ${ }^{2}$ sharmabhavyaa.dav@gmail.com
}

Article History: Received: 11 January 2021; Accepted: 27 February 2021; Published online: 5 April 2021

\begin{abstract}
Data Science and Artificial Intelligence have been all over the world lately,in almost every possible field be it finance,education,entertainment,healthcare,astronomy, astrology, and many more sports is no exception. With so much data, statistics, and analysis available in this particular field, when everything is being recorded it has become easier for team selectors, broadcasters, audience, sponsors, most importantly for players themselves to prepare against various opponents. Even the analysis has improved over the period of time with the evolvement of AI, not only analysis one can even predict the things with the insights available. This is not even restricted to this,nowadays players are trained in such a manner that they are capable of taking the most feasible and rational decisions in any given situation. Chess is one of those sports that depend on calculations, algorithms, analysis, decisions etc. Being said that whenever the analysis is involved, we have always improvised on the techniques. Algorithms are somethingwhich can be solved with the help of various software, does that imply that chess can be fully solved,in simple words does that mean that if both the players play the best moves respectively then the game must end in a draw or does that mean that white wins having the first move advantage. Let's figure it out.
\end{abstract}

\section{Introduction}

Chess is a two-player intellectual, tactical and strategical game played on a 64 squared $8 \times 8$ checkered board, wherein both the players start with 16 pieces, i.e., 8 pawns, 2 knights, 2 bishops, 2 rooks, 1 queen and, 1 king respectively, each piece has its own role to play. Both the players have alternative turns starting from white, moving only one piece at a time, except castling, the ultimate goal of the players should be to checkmate the opponent's king. However, a game can end in a different manner too, say a draw [7].

Parallelly, chess can be played in many variants; according to research,there are more than two thousand published chess variants. Most of them are from recent ones.

The constant evolvement of AI and the involvement of AI in Chess is something to look out for as it is improving day by day. There are several software and machines developed over the period of time to analyze the game. Not only they show the best move but also the alternative considerable moves with their respective evaluation of the chess position and the change in lines(variations) merely by changing a move, not only the move but the move order too, a small mouse slip or misplay reflects a significant change in the evaluation of the chess position.

Chess, Engines (software) and Chess Engines has shown remarkable growth over the years, the improvement rate is so strong that it is speculated that; could any engine will ever be able to solve chess. Though different engines have different capabilities and strengths.

Some of the examples of chess engines and machines are as follows:

1)Stockfish

2)Komodo

3)Leela Chess Zero

4)Houdini

5)AlphaZero

6)Rybka

7)Fritz

8)Shredder and many more [8]

With the help of available data (games, algorithms, chess positions etc) and with the help of the chess engines players nowadays prepare against each other more efficiently. Predicting your opponent has become easier lately.

\section{HISTORY}

Chess is believed to be derived from the Indian game chaturangasometime before the 7 th century. It has evolved over the period of time, there have been many elite players and World Champions; Mikhail Tal, Mikhail Botvinnik, Bobby Fisher, JuditPolgar, VishwanathanAnand, Kasparov, Anatoly Karpov, Magnus Carlsen, Vladimir Kramnik to name a few. 
$1700-1873$

Considered to be the Romantic Era of Chess, there was no engine assistance at that time, players used to create magical games on their own self. Some of the greatest theories of chess were adopted from these games[7].

1945-present

Considered to be the Pre-Modern and Modern Era of chess, a lot had changed with the evolvement of AI and the introduction of chess engines and machines.

In 1989 IBM purchased a computer program and named Deep Thought (now namedDeep Blue) with the sole aim of defeating then World Champion Garry Kasparov. Though it lost its first game against the World Champion, with several improvements in the program and with the passage of time Deep Blue managed to earn its win against the Champion.

There were 6 matches played between the World Champion and the program, the summary of the games is as follows:

Match 1: (1989): Kasparov managed to win this match in forty-five moves by playing King Indian Attack.

Match 2: In Match 2 Deep Blue played the popular Ruy Lopez. The game seemed to be in Kasparov's favor but it turned out really sour unexpectedly. Kasparov claimed the program cheated somehow as it had some sort of knowledge of Champions gameplay.

He immediately resigned, though it was a totally drawn game.

Match 3: Kasparov chose a totally irregular opening assuming that the program must not be having preknowledge of that and the game ended in a draw.

Match 4: Kasparov played very common Caro-Kanndefense. Kasparov played a number of hurried moves, later he had to pay for that as a game totally close to drawing was eventually Deep Blue's win.

Match 5: Kasparov played King's Indian Attack (again) and played very solidly, but the constant improvements in the program led to a very good endgame which was finally a draw.

Match 6: (1997): Kasparov played Caro-Kann (again), but unexpectedly allowed a knight sacrifice from Deep Blues' side and the match went in Deep Blues'favor.Within 20 moves it managed to win. In May 1997, a program officially managed to earn the win against a World Champion.

Ever since the programs and machines are only improving.

\section{APPLICATIONS}

As mentioned earlier chess engines and machines vary in strength, capabilities. We all are familiar with the human versus program thing but it is not restricted to that. Often, we come across tournaments and games where only these engines and machines compete with each other.

Following are some examples of engine vs engine:

CASE 1) When both the programs are of similar strengths.

Say Stockfish and Leela



Position and Evaluation after 11 moves.

This game was played on 11th May 2019, Stockfish (White) opened the game with d4, Leela(black) played King Indian defense. After 143 moves this game ended in a draw. 


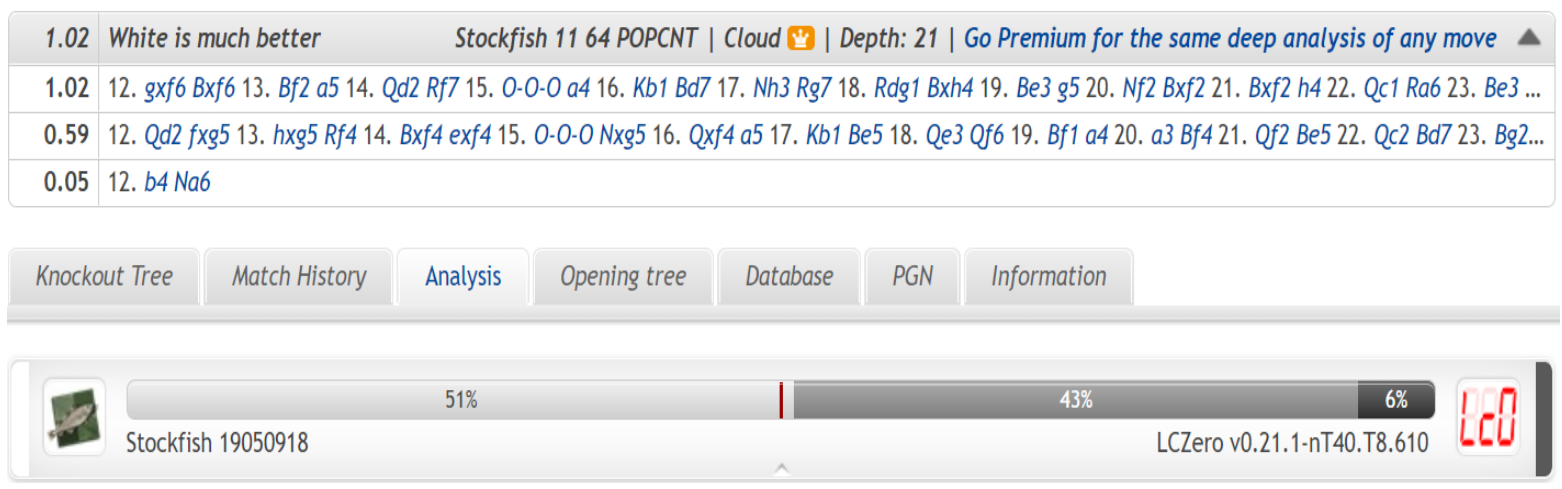

Analysis done by stockfish. Evaluation after 11 moves.

With the help of AI, engines are able to calculate and evaluate different possibilities after every move. In the above picture, various lines(possibilities) are shown after move 11 and the move corresponding to the best evaluation is the best move possible to play in a given situation.

Also provided the Database it is also possible to calculate the win, loss, and draw percentages with the associated openings and lines.

\section{믈 Stockfish 190509183587}

\begin{tabular}{|c|c|c|c|c|c|c|c|c|c|c|c|c|c|c|c|}
\hline $1 / 2$ & $1 / 2$ & $1 / 2$ & $1 / 2$ & $1 / 2$ & $1 / 2$ & $1 / 2$ & $1 / 2$ & $1 / 2$ & 0 & $1 / 2$ & 1 & $1 / 2$ & $1 / 2$ & $1 / 2$ & 0 \\
\hline $1 / 2$ & $1 / 2$ & $1 / 2$ & $1 / 2$ & $1 / 2$ & $1 / 2$ & $1 / 2$ & $1 / 2$ & $1 / 2$ & 1 & $1 / 2$ & 0 & $1 / 2$ & $1 / 2$ & $1 / 2$ & 1 \\
\hline $1 / 2$ & 0 & $1 / 2$ & $1 / 2$ & $1 / 2$ & $1 / 2$ & $1 / 2$ & 0 & $1 / 2$ & 0 & $1 / 2$ & $1 / 2$ & $1 / 2$ & $1 / 2$ & $1 / 2$ & $1 / 2$ \\
\hline $1 / 2$ & 1 & $1 / 2$ & $1 / 2$ & $1 / 2$ & $1 / 2$ & $1 / 2$ & 1 & $1 / 2$ & 1 & $1 / 2$ & $1 / 2$ & $1 / 2$ & $1 / 2$ & $1 / 2$ & $1 / 2$ \\
\hline $1 / 2$ & $1 / 2$ & 1 & 0 & $1 / 2$ & 0 & 1 & 0 & $1 / 2$ & $1 / 2$ & 1 & $1 / 2$ & 1 & $1 / 2$ & $1 / 2$ & $1 / 2$ \\
\hline $1 / 2$ & $1 / 2$ & 0 & 1 & $1 / 2$ & 1 & 0 & 1 & $1 / 2$ & $1 / 2$ & 0 & $1 / 2$ & 0 & $1 / 2$ & $1 / 2$ & $1 / 2$ \\
\hline $1 / 2$ & $1 / 2$ & $1 / 2$ & $1 / 2$ & $1 / 2$ & $1 / 2$ & $1 / 2$ & $1 / 2$ & $1 / 2$ & $1 / 2$ & $1 / 2$ & $1 / 2$ & 0 & 0 & $1 / 2$ & $1 / 2$ \\
\hline $1 / 2$ & $1 / 2$ & $1 / 2$ & $1 / 2$ & $1 / 2$ & $1 / 2$ & $1 / 2$ & $1 / 2$ & $1 / 2$ & $1 / 2$ & $1 / 2$ & $1 / 2$ & 1 & 1 & $1 / 2$ & $1 / 2$ \\
\hline $1 / 2$ & $1 / 2$ & $1 / 2$ & $1 / 2$ & $1 / 2$ & $1 / 2$ & $1 / 2$ & $1 / 2$ & $1 / 2$ & $1 / 2$ & $1 / 2$ & $1 / 2$ & $1 / 2$ & $1 / 2$ & $1 / 2$ & $1 / 2$ \\
\hline $1 / 2$ & $1 / 2$ & $1 / 2$ & $1 / 2$ & $1 / 2$ & $1 / 2$ & $1 / 2$ & $1 / 2$ & $1 / 2$ & $1 / 2$ & $1 / 2$ & $1 / 2$ & $1 / 2$ & $1 / 2$ & $1 / 2$ & $1 / 2$ \\
\hline 1 & 0 & $1 / 2$ & $1 / 2$ & $1 / 2$ & 0 & 1 & 0 & $1 / 2$ & $1 / 2$ & $1 / 2$ & $1 / 2$ & $1 / 2$ & 0 & $1 / 2$ & $1 / 2$ \\
\hline 0 & 1 & $1 / 2$ & $1 / 2$ & $1 / 2$ & 1 & 0 & 1 & $1 / 2$ & $1 / 2$ & $1 / 2$ & $1 / 2$ & $1 / 2$ & 1 & $1 / 2$ & $1 / 2$ \\
\hline $1 / 2$ & $1 / 2$ & $1 / 2$ & $1 / 2$ & $461 / 2$ & & & & & & & & &
\end{tabular}

LCZero v0.21.1-nT40.T8.610 3589

Statistics corresponding to the article published by chess24.com as of June 2019.

Though both the engines are of similar strengths Leela proved to be better than Stockfish in these 100 set games which Leela managed to win by $53.5-46.5$

CASE 2) When both the programs are of different strengths.

Say Stockfish and Rybka 


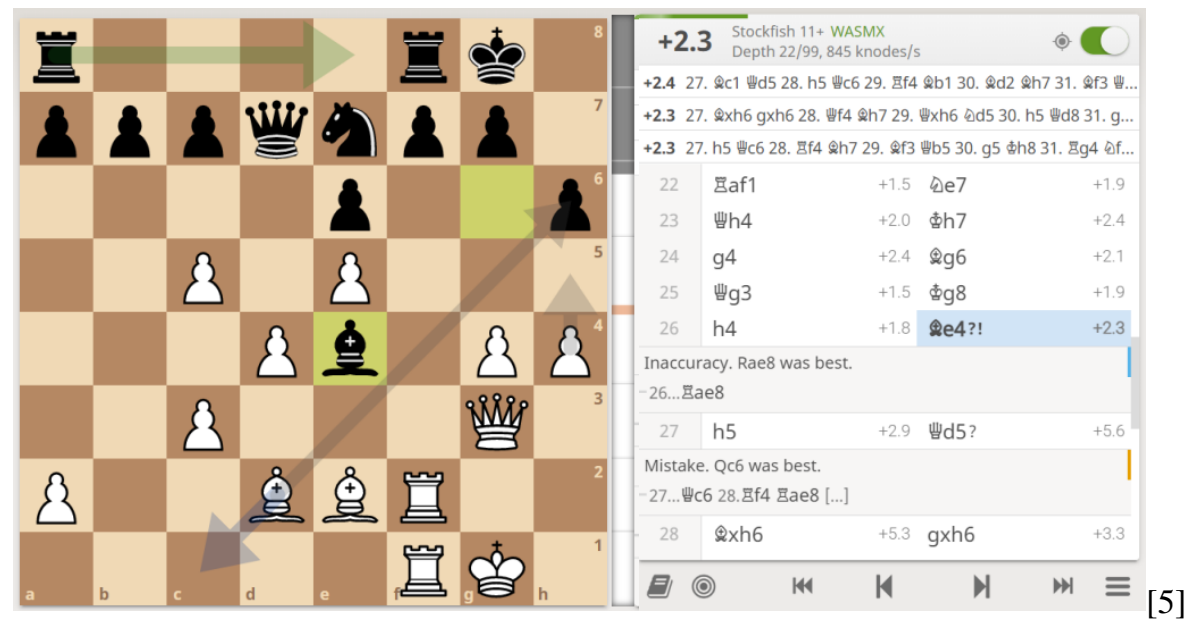

Position and Evaluation after 26 moves.

This game was played on $27^{\text {th }}$ July 2013, Stockfish (White) opened the game with e4,Rybka(black) played the Alekhine defense. Stockfish managed to win in 42 moves.

Again, different lines are calculated and evaluated by the machines and programs shown on the right-hand side of the above picture.

On the left-hand side of the above picture, various arrows are shown, different arrows depict different considerable alternatives of the best move. The best move being the move corresponding to the biggest and darkest arrow in the picture. Again, the size and opacity of the arrow also depict something, all AI, higher the evaluation bigger and darker the arrow is.

\begin{tabular}{|c|c|c|c|c|}
\hline Move & Games & White / Draw / & 3lack & \\
\hline e4 & $1,038,423$ & $33 \%$ & $42 \%$ & $25 \%$ \\
\hline d4 & 826,559 & $34 \%$ & $43 \%$ & $23 \%$ \\
\hline صf3 & 235,288 & $34 \%$ & $44 \%$ & $23 \%$ \\
\hline c4 & 158,750 & $34 \%$ & $43 \%$ & $23 \%$ \\
\hline g3 & 18,614 & $36 \%$ & $39 \%$ & $25 \%$ \\
\hline
\end{tabular}

Calculation of percentage win and draw based on the database provided which opening/movehas been played.

$\begin{array}{ll}\text { Stockfish } \\ 0 & \text { Inaccuracies } \\ 0 & \text { Mistakes } \\ 0 & \text { Blunders } \\ 8 & \text { Average centipawn loss } \\ & \text { rybka } \\ 0 & \text { Inaccuracies } \\ 1 & \text { Mistakes } \\ 0 & \text { Blunders } \\ 24 & \text { Average centipawn loss }\end{array}$

Analysis done by stockfish.

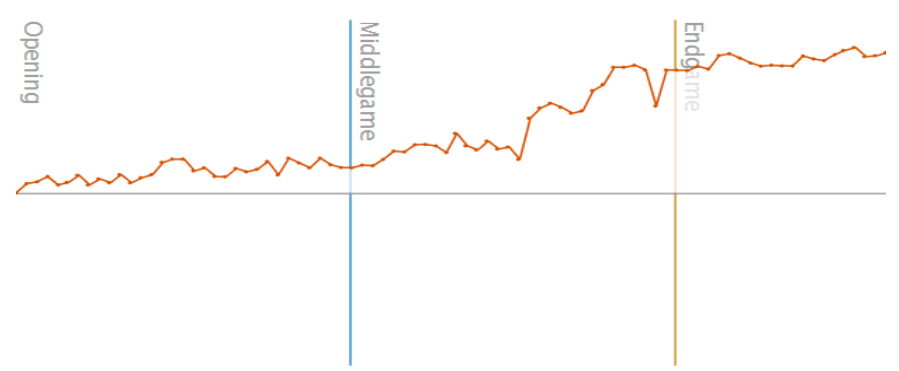

Graphical analysis of above provided Stockfish vs Rybka game. 


\section{Conclusion}

Data Science and Artificial Intelligence have come a long way, there is a lot to explore in a game that has around 1330 openings only, almost 400 board setup possibilities after the $1^{\text {st }}$ move is played by both the players. The number of possible games is too much that one can easily devote a lifetime to that. There have always been mixed views on this topic some even feel that it is already solved. We still need to figure it out There are things that are still needed to be explored.

Conjecture: Considering the ever-improving chess engines and machines that too at a great speed, in my opinion, programs would very soon be able to solve this game. That may be something that programmers want but that is not the thing any day chess players would want.
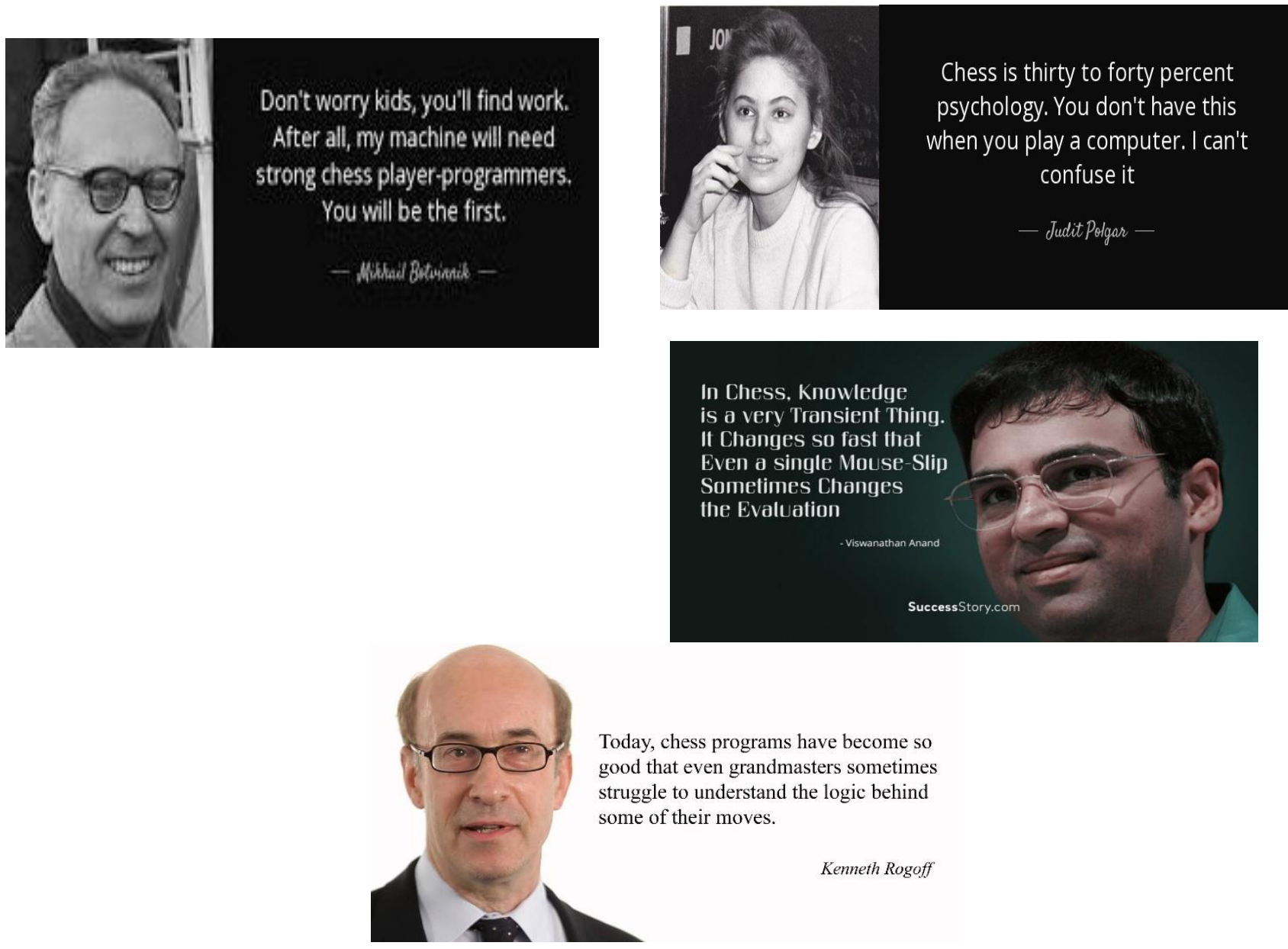

\section{REFERENCES:}

A. https://en.wikipedia.org/wiki/Data_science

B. https://en.wikipedia.org/wiki/Data_analysis

C. https://en.wikipedia.org/wiki/Category:Computational_fields_of_study

D. Chess.com

E. Lichess.org

F. Chess24.com

G. https://en.wikipedia.org/wiki/Chess

H. https://en.wikipedia.org/wiki/Chess_engine

I. https://en.wikipedia.org/wiki/Garry_Kasparov

J. https://www.ibm.com/in-en

K. https://thebestschools.org/magazine/brief-history-of-computerchess/\#: :text=History\%20of\%20Chess $\% 20$ Playing\%20Computers,a $\% 20$ full $\% 20$ game $\% 20$ of $\% 20$ ches s.

L. https://www.chess.com/forum/view/general/will-computers-ever-solve-chess

M. https://www.chessgames.com/perl/chessgame?gid=1717104 


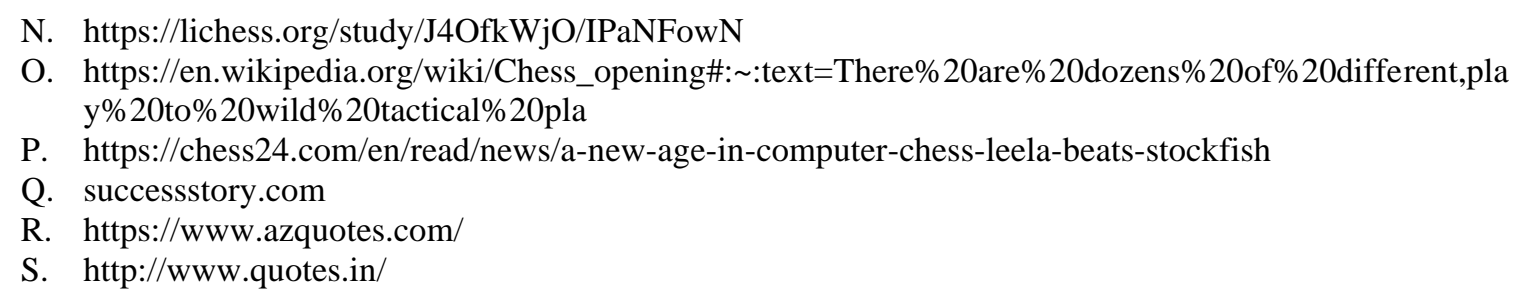

